\title{
NUTRICIÓN Y SALUD PÚBLICA: POLÍTICAS DE ALIMENTACIÓN ESCOLAR EN LA ESPAÑA CONTEMPORÁNEA (1931-1978)
}

\author{
Eva María Trescastro-López, Josep Bernabeu-Mestre y María Eugenia Galiana-Sánchez \\ Grupo Balmis de Investigación en Salud Comunitaria e Historia de la Ciencia. Universidad de Alicante \\ eva.trescastro@ua.es
}

Recibido: 6 agosto 2012; Aceptado: 22 noviembre 2012.

Cómo citar este artículo/Citation: Trescastro-López, Eva María; Bernabeu-Mestre, Josep y Galiana-Sánchez, María Eugenia (2013), “Nutrición y salud pública: políticas de alimentación escolar en la España contemporánea (1931-1978)", Asclepio 65 (2): p026. doi: http://dx.doi.org/10.3989/asclepio.2013.26

\begin{abstract}
RESUMEN: El trabajo aborda los antecedentes históricos de las políticas de alimentación escolar en la España del siglo XX, a través del análisis del corpus normativo y de los textos y materiales que generaron organismos nacionales e internacionales que participaron en su implementación. La investigación muestra el impacto que tuvo el contexto internacional y en particular los acuerdos que se alcanzaron durante el primer franquismo con los Estados Unidos y con la FAO y UNICEF, en el desarrollo de dichas políticas. El carácter filantrópico de las primeras cantinas fue sustituido por una creciente implicación del ámbito público, al incorporar la alimentación de los escolares como objetivo de las políticas sanitarias y educativas de la Segunda República. La Ley de Educación Primaria de 1945 permitió la gradual institucionalización de la alimentación escolar. La creación en 1954 del Servicio Escolar de Alimentación y Nutrición y la necesidad de coordinar la ayuda norteamericana facilitaron la implementación de programas como el de Productos Lácteos pro Bienestar Infantil y Social o el de Educación en Alimentación y Nutrición, lo cual permitió implantar el complemento alimenticio en escolares y llevar a cabo un conjunto de actividades educativas coordinadas encaminadas a mejorar su alimentación.
\end{abstract}

PALABRAS CLAVE: Nutrición; Salud Pública; Políticas de nutrición; Alimentación escolar; Historia del siglo XX; España.

NUTRITION AND PUBLIC HEALTH: SCHOOL NUTRITION POLICIES IN CONTEMPORARY SPAIN (1931-1978)

ABSTRACT: This paper examines the historical background of school nutrition policies in twentieth-century Spain, by analysing the body of regulations and the texts and materials produced by the national and international organisations involved in their implementation. The research reveals the influence and impact of international relationships and in particular of the agreements reached during the early years of the Franco regime with the United States and FAO and UNICEF in the development of nutrition policies. The philanthropic nature of school canteens gave way to the increasing involvement of the public sector and the provision of school nutrition became one of the basic objectives of the health and education policies of the Second Republic. The Primary Education Act of 1945 provided the legal framework for the gradual institutionalisation of school nutrition. The creation in 1954 of the School Food and Nutrition Service, together with the need to coordinate U.S. aid, facilitated the implementation of programmes such as Dairy Products for Child and Social Welfare or Food and Nutrition Education, it was through these programmes that school meals for children were established and a set of coordinated educational activities aimed at improving school children's diet and nutrition were implemented.

KEY WORDS: Nutrition; Public Health; Nutrition Policy; School Feeding; History of $20^{\text {th }}$ Century; Spain.

Copyright: (c) 2013 CSIC. Este es un artículo de acceso abierto distribuido bajo los términos de la licencia Creative Commons Attribution-Non Commercial (by-nc) Spain 3.0. 


\section{INTRODUCCIÓN}

Una correcta alimentación repercute positivamente en la salud y en la población infantil despierta un especial interés ya que constituye un elemento fundamental para su completo desarrollo (Rigolfas, Padró y Cervera, 2010).

En el año 2005 el Ministerio de Sanidad y Consumo puso en marcha la Estrategia NAOS (Nutrición, Actividad Física y Prevención de la Obesidad) para sensibilizar a la población del problema de la obesidad e impulsar iniciativas que contribuyan a lograr que los ciudadanos, y especialmente niños y jóvenes, adopten hábitos de vida saludables, principalmente a través de una alimentación equilibrada y de la práctica de actividad física (Agencia Española de Seguridad Alimentaria, 2005). Bajo las directrices de la Estrategia NAOS, en el año 2006 se puso en marcha el programa piloto PERSEO, para promover la adquisición de hábitos alimentarios saludables, estimular la práctica de actividad física entre los escolares y prevenir la aparición de obesidad y otras enfermedades. Sin embargo, como se podrá comprobar, en el caso español existían importantes antecedentes en materia de políticas relacionadas con la alimentación de los escolares.

El objetivo de este trabajo es, en primer lugar, analizar la evolución de las iniciativas de alimentación escolar que se pusieron en marcha en España a lo largo del siglo XX y el contexto en el que se implantaron. En segundo lugar, mostrar la importancia de tomar en consideración la experiencia histórica en el momento de diseñar y establecer políticas alimentarias. Para ello, junto al análisis de la legislación promulgada durante el período 1900-1978, se han utilizado sobre todo los textos y materiales que generaron el Programa de Educación en Alimentación y Nutrición (EDAL$\mathrm{NU}$ ) y organismos nacionales como el Servicio Escolar de Alimentación y Nutrición (SEAN) o internacionales como la FAO.

\section{EL ANTECEDENTE DE LAS CANTINAS ESCOLARES EN LAS PRIMERAS DÉCADAS DEL SIGLO XX}

A finales del siglo XIX y las primeras décadas del siglo XX, el movimiento higienista español, al igual que ocurrió en el ámbito internacional, avanzó en el desarrollo del concepto de «infancia protegible» (Perdiguero, 2004; Barona, 2006). Al mismo tiempo que tenía lugar un creciente interés sanitario por los problemas relacionados con la alimentación y la nutrición de la población (Bernabeu-Mestre et al., 2011; Bernabeu-Mestre, Trescastro-López y Galiana-Sánchez, 2011), primero desde el ámbito de la filantropía y con posterioridad desde la administración sanitaria y educativa, se fueron configurando iniciativas y políticas encaminadas a conseguir que la alimentación y nutrición de los escolares fuera adecuada en función de su edad y necesidades fisiológicas (Scott, 1954).
Como ocurría en el contexto internacional (Hendrich, 1992; Barona, 2012) en España, muchas de esas iniciativas se tradujeron en la creación de cantinas y colonias escolares, algunas mantenidas por asociaciones como la de Caridad Escolar, la del Desayuno Escolar o la del Niño Descalzo, entre otras (TrescastroLópez, Bernabeu-Mestre y Galiana-Sánchez, 2011)ํ․ Conocidas en el ámbito de la pedagogía como «instituciones circumescolares» tenían una doble misión (Hernández, Hernández y Sanromà, 2005). Por un lado, proporcionaban una alimentación que fuera "sana y abundante», para cubrir las necesidades de los niños ${ }^{2}$ y por otro se aprovechaba su potencial educativo. La educación no solo se limitaba al aspecto alimentario, sino también al «higiénico y moral», contribuyendo a la adquisición de «buenos modales y hábitos de orden y limpieza».

Tal fue el interés que despertaron estas iniciativas filantrópicas, que en 1912 el Ministerio de Instrucción Pública y Bellas Artes, además de reconocer su labor ${ }^{3}$, estimó conveniente reunir los datos referentes a su organización y funcionamiento para conocer los resultados y analizar sus objetivos, aunque finalmente no se llegó a publicar dicha información ${ }^{4}$.

En 1921 se crearon 3 plazas específicas de maestras para reforzar la labor de la cantina del Grupo Escolar Cervantes de la Escuela Graduada de Magisterio de Madrid $^{5}$. En las décadas de 1920 y 1930, desde el Ministerio se incoaron diversos expedientes que permitieron clasificar algunas cantinas como «Asociación benéfico-docente de carácter particular», tal como ocurrió con el Patronato de Cantinas y Colonias Escolares de la Coruña, o la Cantina Escolar de Benavente, entre otras ${ }^{6}$. Así mismo, en el marco de las campañas institucionales destinadas a abordar el problema sociosanitario de las Hurdes (Sánchez-Granjel, 2003), se concedió una cantidad de 10.000 pesetas para la implantación de cantinas escolares ${ }^{7}$.

\section{LAS INICIATIVAS DE LA 2a REPÚBLICA}

Tras una primera etapa donde la iniciativa filantrópica fue esencial para la creación, mantenimiento y funcionamiento de las cantinas, se produjo su progresiva asunción pública. Las cantinas modificaron su concepción de instituciones benéficas y en 1931, el Gobierno republicano socialista, de acuerdo con sus políticas en materia educativa y sanitaria (Barona y Bernabeu-Mestre, 2008), decidió integrarlas en la obra educativa de la escuela y decretó que los consejos locales de primera enseñanza crearan cantinas en cuantas escuelas fuera posible y se sostuvieran con las subvenciones del Estado y municipios, además de donativos, colectas y suscripciones ${ }^{8}$. Aunque se continuó reconociendo la condición de "Asociación benéfico-docente de carácter particular ${ }^{9}$, desde el ámbito 
gubernamental se realizó un importante esfuerzo presupuestario para su mantenimiento. El capítulo destinado a dichas partidas, pasó de 450.000 pesetas en 1931 a 2,7 millones de pesetas en $1934^{10}$ y alcanzó la cifra de 7 millones en $1937^{11}$.

Por otra parte, en un intento por regular su actividad y conseguir sus objetivos, se intensificó la reglamentación sobre las cantinas. Para que las subvenciones llegaran al mayor número posible se creó en el Ministerio de Instrucción Pública y Bellas Artes una Comisión Central de Colonias, Cantinas y Roperos Escolares, encargada de la distribución del presupuesto, de normativizar la concesión de ayudas y de inspeccionar que cumplieran su "alta función educadora» ${ }^{12}$.

En 1935 el Ministerio resolvió que en toda escuela nacional de primera enseñanza habría de existir una comisión protectora y los inspectores de primera enseñanza debían comprobar, no solo la inversión de los fondos, sino también el programa de alimentación y la selección de alumnos beneficiarios. En consonancia con el desarrollo que estaba alcanzando la nutrición comunitaria (Bernabeu-Mestre, et al., 2011, p.193), la supervisión sanitaria de las cantinas recayó en los médicos de asistencia pública domiciliaria, en aquellos casos en los que no existía la figura del inspector médico escolar de distrito (Ballester, 1994). Además, aquellas cantinas escolares que venían funcionando a cargo de "Asociaciones, Juntas o Comisiones», pasaron a integrarse en el organigrama de control y supervisión ${ }^{13}$.

Estas iniciativas encaminadas a conseguir la institucionalización pública del modelo de cantinas se vieron afectadas por la guerra civil, período durante el cual únicamente se otorgaron subvenciones para organización y mantenimiento de servicios básicos ${ }^{14}$, dotación de enseres y menaje imprescindible ${ }^{15}$ y para hacer frente a los gastos de alimentación de la infancia evacuada ${ }^{16}$

\section{LA ALIMENTACIÓN ESCOLAR DURANTE EL FRAN- QUISMO}

La alimentación escolar durante el franquismo tuvo un antes y un después con la promulgación de la Ley de Educación Primaria de 1945. Durante los primeros años de posguerra se produjo un importante deterioro nutricional de la población (Del Cura y Huertas, 2007); sin embargo, aunque el Estado continuó subvencionando con cantidades variables las cantinas, el presupuesto asignado disminuyó en relación con el período republicano ${ }^{17}$ (Figura 1).

El 17 de julio de 1945 se promulgó la Ley de Educación Primaria, y en ella se intentó recoger las iniciativas que tenían como objetivo proteger a la infancia. En su artículo 12 se especificaba: "la enseñanza obligatoria llevará consigo la debida protección para aquellos escolares que por su pobreza no pudieran concurrir a las Escuelas sin asistencia de alimentación y vestido». En el artículo 47 del capítulo V, en el apartado dedicado a las actividades complementarias a la escuela, se detallaba que en este grupo quedaban comprendidas cuantas instituciones tuvieran por misión el cumplimiento del artículo 12 y declaraba:

para alimento y vestido de los niños se crea en todas las Escuelas públicas (...) el servicio de comedores y roperos escolares. Los niños pudientes que utilizaren el comedor o el ropero escolar abonarán el importe que corresponda (...). Los niños que carezcan de recursos disfrutarán del servicio gratuito, y su sostenimiento corresponderá, en todo o en parte, al Estado y a las Corporaciones públicas $(. . .)^{18}$.

Fue a partir de este momento, cuando la gestión, coordinación y mantenimiento de las cantinas o comedores pasó a ser asumida plenamente por la iniciativa pública.

Figura 1. Importe destinado a cantinas y comedores escolares

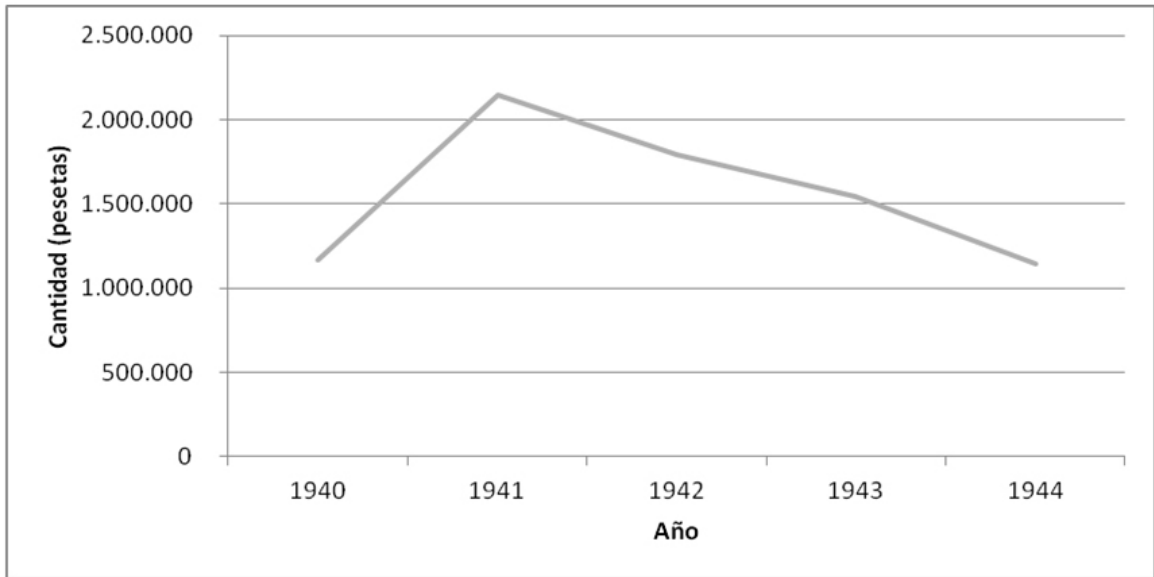

Fuente: Elaboración propia a partir de los datos publicados en el BOE. 
Como consecuencia del desarrollo de dicha Ley, en 1952 se creaba el "Servicio de Protección de la Alimentación Escolar Infantil» (tabla 1) con el objeto de «perfeccionar en todos los aspectos la nutrición de los alumnos de las escuelas nacionales y de orientar debidamente los comedores escolares sostenidos por el Ministerio de Educación Nacional, así como instruir a las familias en orden a la alimentación de sus hijos» ${ }^{19}$.

Dos años más tarde, en 1954 (tabla 1), se firmó un convenio entre el Fondo de las Naciones Unidas para la Infancia (UNICEF) y el Gobierno español, y se organizó un sistema de ayudas destinadas a niños, adolescentes, mujeres embarazadas y lactantes. Dicho convenio permanecería en vigor durante el tiempo necesario para que los planes de operaciones pudieran llegar a ejecutarse ${ }^{20}$. Ese mismo año se creó la
Comisión Interministerial para el Auxilio Internacional a la Infancia ${ }^{21}$ (CIAII) en el Ministerio de Asuntos Exteriores (tabla 1). La CIAll sería la encargada de la ejecución práctica del convenio, la preparación y elaboración de los planes de operaciones y la utilización y distribución de los artículos que el Fondo facilitara. Fruto de la labor de la CIAII, a lo largo de las décadas de 1950 y 1960 se fueron aprobando e implantando diversos programas nacionales: el de productos lácteos pro bienestar infantil y social; el de educación en alimentación y nutrición (EDALNU), el de sanidad (prematuros, rehabilitados, tracoma, etc.), el de ayuda social americana, etc., todos relacionados con los planes de operaciones suscritos con los Organismos Internacionales UNICEF, FAO y la National Catholic Welfare Conference (NCWC) ${ }^{22}$.

Tabla 1. Resumen de las principales iniciativas y acontecimientos relacionadas con alimentación escolar en España, 1952-1978

\section{2}

Se crea el Servicio de Protección de la Alimentación Escolar Infantil

\section{4}

Se firma convenio entre Fondo Naciones Unidas para la Infancia y Gobierno Español

Se crea la CIAlla en el Ministerio de Asuntos Exteriores encargada de la ejecución de los principios del convenio y la puesta en marcha de diversos programas nacionales

1959

Se pone en funcionamiento el Programa de Productos Lácteos pro Bienestar Infantil y Socia

1961

\begin{tabular}{|c|c|c|}
\hline \multicolumn{3}{|c|}{1961} \\
\hline \multicolumn{3}{|c|}{ Se pone en funcionamiento el Programa EDALNU ${ }^{c}$} \\
\hline \multicolumn{3}{|c|}{1962} \\
\hline Se convoca $1^{\circ}$ curso Diplomados EDALNU & $\begin{array}{l}\text { Los fondos de Programa EDALNU pasan del } \\
\text { Ministerio de Exteriores al Ministerio de } \\
\text { Hacienda }\end{array}$ & $\begin{array}{l}\text { Se crean Escuelas de Educación en Alimentación } \\
\text { y Nutrición en régimen de Consejo Escolar } \\
\text { Primario }\end{array}$ \\
\hline \multicolumn{3}{|c|}{1965} \\
\hline Se convoca 20 curso Diplomados EDALNU & \multicolumn{2}{|c|}{$\begin{array}{l}\mathrm{ClAll} \text { se transforma en } \mathrm{ClBIS}^{\mathrm{d}} \text { manteniendo las mismas funciones y queda asignada al Ministerio de } \\
\text { la Gobernación }\end{array}$} \\
\hline \multicolumn{3}{|c|}{1973} \\
\hline $\begin{array}{l}\text { CIBIS adscribe sus programas (entre ellos EDALNU) a } \\
\text { la Dirección General de Política Interior y Asistencia } \\
\text { Social }\end{array}$ & \multicolumn{2}{|c|}{$\begin{array}{l}\text { Se disuelven las Escuelas de Educación en Alimentación y Nutrición en régimen de Consejo Escolar } \\
\text { Primario }\end{array}$} \\
\hline \multicolumn{3}{|c|}{1978} \\
\hline
\end{tabular}

CIBIS transfiere todas sus funciones al FNAS y los Programas (entre ellos EDALNU) pasan a depender de la Dirección General de Salud Pública y Sanidad Veterinaria (Gestora del FNAS)

a. Comisión Interministerial para el Auxilio Internacional a la Infancia

b. Servicio Escolar de Alimentación y Nutrición

c. Programa de Educación en Alimentación y Nutrición

d. Comisión Interministerial pro Bienestar Infantil y Social

e. Fondo Nacional de Asistencia Social 
También en 1954 (tabla 1), se creaba el Servicio Escolar de Alimentación y Nutrición (SEAN) dependiente de la Dirección General de Enseñanza Primaria con el objetivo de poner en práctica el artículo 47 de la Ley de Educación Primaria. Entre las funciones del SEAN destacaba la misión de organizar, distribuir e instalar los medios necesarios para el establecimiento del complemento alimenticio y cooperar en la orientación dietética en la edad escolar ${ }^{23}$. EI SEAN tenía una doble misión, por un lado asegurar a los alumnos que acudían a los comedores y colonias una alimentación adecuada e influir, a través del niño, en la educación sanitaria de la familia (Servicio Escolar de Alimentación, 1955, p. 13). Por otro, coordinar las políticas e iniciativas nacionales y la ayuda internacional. Se trataba de un conjunto de actividades en las que estaban implicados diversos ministerios y direcciones generales (Vivanco, 1965; Trescastro-López y TrescastroLópez, 2013).

En diciembre del mismo año ${ }^{24}$ se amplió la composición y funciones de la CIAll al extender sus competencias a la distribución de los suministros que facilitara a «Caritas» la NCWC. En 1955 se publicaron las normas ejecutivas para el desarrollo de la «Ayuda Social Americana para los económicamente débiles», incluida en los acuerdos bilaterales firmados con EEUU en 1953 (Barciela, 2000), cuyos objetivos eran la intensificación en España de los servicios de cantinas escolares; el fomento de la alimentación complementaria para tuberculosos, madres gestantes y lactantes y niños menores de tres años; el suministro de la ayuda a establecimientos benéficos y de asistencia sanitaria y social; y el mantenimiento de la asistencia social y domiciliaria practicada por Cáritas y la Obra Social de la Sección Femenina ${ }^{25}$. La alimentación complementaria contemplaba el reparto de productos lácteos y du- rante el curso 1955-1956 se implantó en las escuelas españolas. Aunque ya existían investigaciones sobre los beneficios que aportaba dicha suplementación en escuelas inglesas, francesas o estadounidenses (Scott, 1954 , p. 22), se pusieron en marcha en España estudios en los que se comprobaba el aumento de peso y talla con la suplementación de $250 \mathrm{ml}$ de leche, 10 g mantequilla y $30 \mathrm{~g}$ de queso por ración a niños de grupos económicamente débiles (Altava, 1957, p. 30; Vivanco, Palacios y García, 1984, pp. 34-38).

El Gobierno español, además de distribuir la Ayuda Social Americana, se comprometía a mejorar el nivel de vida de las clases necesitadas, de manera que conforme la ayuda internacional se fuera reduciendo se «debían incrementar los fondos nacionales» ${ }^{26}$. También se incentivó la producción de leche convocando concursos entre empresas españolas para la adjudicación del suministro de leche destinada al programa sanitario-pedagógico-docente desarrollado por el SEAN ${ }^{27}$.

Entre los años 1955 y 1966 la administración española, además de las subvenciones aprobadas en los presupuestos generales, destinó más de 551.464.626 pesetas como créditos extraordinarios para los gastos originados por la distribución de los productos de la Ayuda Social Americana ${ }^{28}$ (figura 2).

En 1959 se creó, dependiente de la CIAll, la Comisión Mixta de Productos Lácteos pro Bienestar Infantil y Social (tabla 1), encargada de ejecutar el programa del mismo nombre derivado del convenio de 1954, que estaba regido por un órgano ejecutivo de Gerencia radicado en el SEAN ${ }^{29}$. Ambas Comisiones, la Interministerial y la Mixta fueron ampliando su composición con el fin de implicar al mayor número posible de organismos ${ }^{30}$.

Figura 2. Gastos extraordinarios originados por la distribución de productos alimenticios de la Ayuda Social Americana

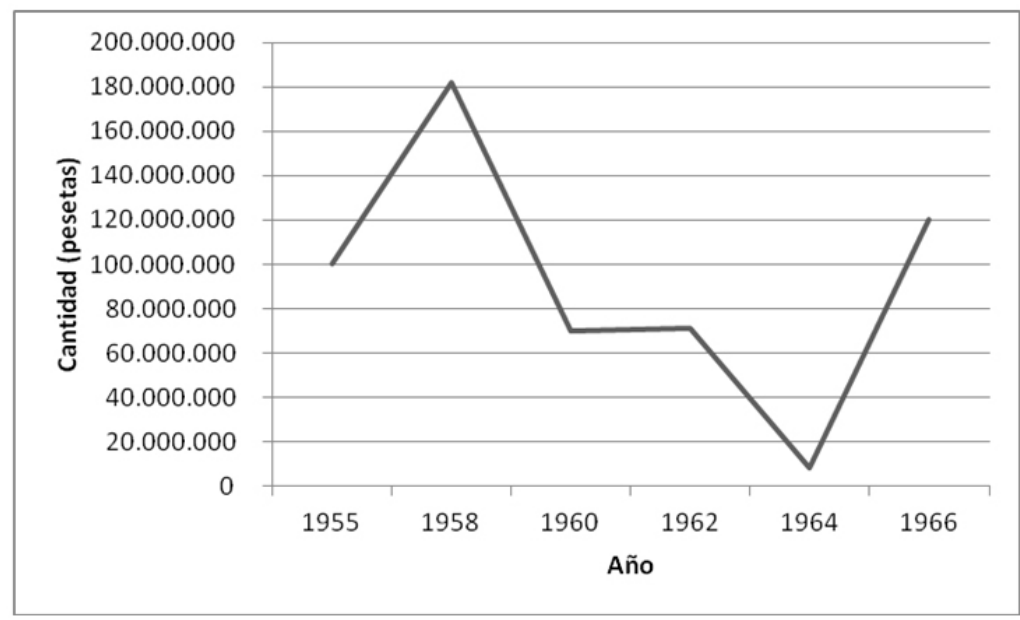

Fuente: Elaboración propia a partir de los datos publicados en el BOE. 
Unos años después, en 1961, se ponía en marcha el Programa EDALNU (De Palacios, 1969; López, 1972; López, 1973; López, 1987; García y López, 1990) (tabla 1), cuyo objetivo principal era mejorar el nivel nutricional de la familia española introduciendo la educación en nuevos y mejores hábitos alimentarios y el estímulo para la producción y consumo de alimentos locales (Esplugues-Pellicer y Trescastro-López, 2012; Trescastro-López, Galiana-Sánchez y Bernabeu-Mestre, 2012; Trescastro-López y Trescastro-López, 2013). El Programa fue asignado a la Dirección General de Enseñanza Primaria a través del SEAN, «en estrecho contacto» con las Direcciones Generales de Sanidad, de Coordinación, Crédito y Capacitación Agraria, y con la Sección Femenina de FET y de las JONS, así como con los Organismos internaciones FAO y UNICEF. Para poder desarrollar los objetivos del Programa, se convocó en 1962 el primer curso de Diplomados en Educación en Alimentación y Nutrición (EDALNU) (tabla 1) para capacitar al personal que debía difundirlo. Dicho curso se celebró en Madrid y participaron quince profesoras enfermeras puericultoras, diez miembros del Servicio de Extensión Agraria, once Instructoras de la Sección Femenina y cincuenta y seis educadores ${ }^{31}$. Cabe resaltar a los maestros entre el colectivo de Diplomados EDALNU; para favorecer su labor y continuidad se creó un Consejo Escolar Primario compuesto por 56 Escuelas Nacionales de Educación en Alimentación y Nutrición: tres en Madrid; dos en Barcelona, Oviedo, León y Valencia; y una en cada una de las restantes provincias, con excepción de las africanas. Al frente de dichas Escuelas estuvieron maestros nacionales en posesión del Diploma EDALNU encargados de desarrollar el Programa en el ámbito escolar ${ }^{32}$.

En 1963 ante el desarrollo del SEAN y para facilitar la continuidad del Programa EDALNU en las escuelas, se crearon cuatro departamentos ${ }^{33}$ : el de Educación en Alimentación y Nutrición a cargo de Doña Felisa de las Cuevas Canilla; el de Complemento Alimenticio, a cargo de Don José Matesanz Sanz; el de Comedores Escolares, a cargo de Doña Dolores Prados Beltrán ${ }^{34}$; y el de Programación, a cargo de Don Manuel Rivas Navarro.

Durante los años siguientes se produjo una disminución de la aportación internacional a la alimentación escolar y se incrementó la contribución española, como consecuencia del «progresivo aumento del nivel de ingresos públicos del Estado a causa del proceso de desarrollo económico y social» ${ }^{35}$. En 1965 la CIAll fue sustituida por la Comisión Interministerial pro Bienestar Infantil y Social (CIBIS) (tabla 1), dependiente del Ministerio de la Gobernación, al haberse incrementado la implicación nacional en los programas. Esta Comisión sucesora de la anterior se hacía cargo de los servicios, bienes, derechos y obligaciones de aquella ${ }^{36}$. Ese mismo año se convocó el II Curso de
Diplomados EDALNU para maestros nacionales ${ }^{37}$ para profundizar en el desarrollo del Programa.

Entre las primeras funciones llevadas a cabo por los Diplomados EDALNU estuvo la evaluación del estado nutricional de la población, a través de encuestas mediante el sistema de inventarios y compras y el estudio de la talla de la población española de 4 a 14 años (Vivanco, Palacios y García, 1984, pp.34-38; García y López, 1990)38. Se analizaron 76 municipios de la Península y archipiélagos, lo que permitió obtener el patrón de crecimiento de los niños españoles y se realizó una educación alimentaria para la población general. En 1966 habían recibido algún tipo de capacitación 8.222 personas, existían 5.740 centros con comedor escolar, 404 huertos escolares, 110 granjas escuelas y 432 "clubes escolares» (Casado, 1967, p. 196; Bernabeu-Mestre, et al., 2011, p. 207; Trescastro-López y Trescastro-López, 2013).

En el año 1973 (tabla 1) la CIBIS adscribió la dirección de sus programas a la Dirección General de Política Interior y Asistencia Social ${ }^{39}$. Ese mismo año se disolvió el Consejo Escolar Primario constituido por las Escuelas de Educación en Alimentación y Nutrición transformándolas en unidades escolares de régimen ordinario que se debían integrar en los colegios nacionales $^{40}$. En 1978 (tabla 1), la CIBIS desapareció al coincidir sus funciones con las del Fondo Nacional de Asistencia Social (FNAS) al que fueron transferidos sus recursos financieros, funciones, programas, servicios, bienes, derechos y obligaciones ${ }^{41}$. Finalmente ese mismo año se aprobó el Plan de Inversiones del FNAS y el «Programa de Promoción [sic] de la Educación en Alimentación y Nutrición» (EDALNU) pasó a depender de la Dirección General de Salud Pública y Sanidad Veterinaria como entidad gestora del FNAS ${ }^{42}$.

Como se ha podido comprobar, las iniciativas relacionadas con políticas de alimentación escolar culminaron con el Programa EDALNU y su contribución a las políticas de promoción de la salud. En relación con la valoración de su impacto, hay que recordar que los hábitos alimentarios están influidos por una gran variedad de factores, por lo que atribuir las modificaciones de los mismos a un único elemento de forma independiente resulta extremadamente complejo, tal como indican los expertos en educación alimentaria (López et al., 1998, p. 97). La evaluación de este tipo de intervenciones educativas puede realizarse por diversas vías: presencia de alimentos en el mercado antes inexistentes, aumento del consumo de determinados alimentos, o también a través de indicadores como el número de acciones, cantidad de personal formado o materiales que genera una iniciativa. En este sentido, a lo largo de su desarrollo, el Programa EDALNU realizó más de 100 encuestas en todo el territorio nacional para conocer el estado nutricional de 
la población española y sus hábitos alimentarios, se produjeron y distribuyeron gratuitamente más de 30 millones de publicaciones: libros, folletos, manuales, diapositivas, carteles, etc., y se llevaron a cabo cursos de formación a 3.000 Diplomados y 45.000 Iniciados EDALNU aproximadamente. Durante el tiempo de desarrollo del programa su presencia en los medios de comunicación (radio, prensa y TV) fue abundante, y se difundieron mensajes educativos a la población en general para grupos vulnerables. Además, el Programa EDALNU diseñó la primera guía alimentaria española: la Rueda de los 7 Grupos de Alimentos. Igualmente, en las Escuelas Primarias se realizaron diferentes encuestas y se valoró el impacto del Programa a través de los conocimientos alcanzados por los niños, obteniéndose unos resultados de conocimientos satisfactorios en el $70-80 \%$ de los casos. Al mismo tiempo tenía lugar una mejora de la oferta de los planes de dietas de la red Nacional de Comedores Escolares (López et al., 1998, pp. 96-97).

También se analizaron los datos de tendencias de consumo de alimentos en la población y se estudiaron comparativamente estadísticas de consumo de alimentos facilitadas por el Ministerio de Agricultura desde 1962 hasta 1968, mostrando un aumento en el consumo de leche, productos lácteos, carnes, huevos, frutas y azúcares. El consumo de patatas y de pan disminuía, mientras que no se modificó el consumo de pescado, legumbres, verduras, arroz y grasa. El Programa EDALNU pudo contribuir a corregir algunas deficiencias, como ocurría con la leche o las frutas, pero no pudo evitar que se empezaran a dibujar pautas de consumo poco saludables como las que han caracterizado la transición nutricional española en las décadas finales del siglo XX (López, 1972, p. 956; Serra-Majem y Bautista-Castaño, 2007).

\section{CONCLUSIONES}

Este trabajo ha permitido visualizar la progresiva implementación de las políticas de alimentación escolar a lo largo del siglo XX y la influencia e impacto que tuvo el contexto internacional, como ocurrió con los acuerdos que se firmaron durante el primer franquismo con Estados Unidos o con organismos como FAO y UNICEF.

Aunque en trabajos previos se han analizado algunas de las iniciativas que generaron las políticas de alimentación escolar, los resultados de esta investigación han permitido, en primer lugar, completar una visión de conjunto. En segundo lugar, mostrar la necesidad de profundizar en el abordaje de las actividades que llevaron a cabo organismos como el SEAN o el impacto que tuvo sobre la salud de la población escolar el complemento alimenticio.

La investigación ha mostrado el interés de conocer la experiencia histórica, reflexionar sobre ella a la hora de diseñar las actuales políticas de alimentación y nutrición, y contemplar dichos antecedentes en la elaboración del corpus legislativo y normativo que se está desarrollando en el ámbito del Estado español. A pesar de los resultados alcanzados en las décadas de 1960-1970, hubo que esperar hasta la puesta en marcha de la Estrategia NAOS en el año 2005 para recuperar políticas de nutrición capaces de implicar al gran número de organismos necesario para permitir el desarrollo de programas de nutrición aplicada, como el EDALNU. Se trataría de aprovechar los resultados y experiencias de los programas que se implementaron en el pasado, tal como ocurrió con actividades tan innovadoras en aquel momento como los huertos, las granjas o los clubes escolares que contemplaba el Programa EDALNU.

\section{AGRADECIMIENTOS}

Este trabajo se ha desarrollado en el marco de los proyectos de investigación: "La lucha contra la desnutrición en la España contemporánea y el contexto internacional (1874-1975)" (Ref: HAR200913504-C02-01), Ministerio de Ciencia e Innovación; Beca Predoctoral del Programa de Formación de Profesorado Universitario (Ref: FPU/AP2008-03309), Ministerio de Educación; Programa Prometeo de la Generalitat Valenciana (Ref: Prometeo/2009/122); proyecto "Género, Ideología y Salud: la Enfermería de Salud Pública en la Sanidad Española Contemporánea (1923-1959)" Ref: GRE10-12 del Vicerrectorado de Investigación de la Universidad de Alicante; proyecto "Género, Ideología y Salud: la Enfermería de Salud Pública en la Sanidad Española Contemporánea (19231959)" Ref: GV/2012/100 de la Conselleria de Educació de la Generalitat Valenciana y el proyecto "La sanidad internacional y la transferencia de conocimiento científico en Europa 1900-1975". Período: 2011-2013 (Ministerio español de Economía y Competitividad HAR2011-23233). Agradecemos al profesor Carlos Barciela y a la profesora Mercedes Pascual su colaboración, comentarios y sugerencias. 


\section{NOTAS}

1 Al respecto se publicaron varias memorias de asociaciones como: Asociación del Desayuno Escolar (1917), Memoria aprobada por la Comisión permanente de la citada Institución Benéfica el día 7 de enero de 1917, Madrid, El reformista pedagógico; o también Asociación del Niño Descalzo (1907), El niño Descalzo: sociedad de protección a la infancia, La Coruña, Imprenta de la casa de Misericordia.

2 Este mensaje aparecía en todas las memorias de la Asociación de Caridad Escolar (1905-1927), Memorias aprobadas por la Junta General de la Asociación de Caridad Escolar en las anualidades comprendidas entre 1905-1927, Madrid.

3 Véase R.O. de 22 de marzo de 1912 por la que se le da las gracias a las alumnas de la Escuela Normal Central de Maestras, Gaceta de Madrid, no 88, (28-03-1912).

4 Véase R.O. de 30 de diciembre de 1911 por la que se estima necesario reunir los datos referentes a la organización y funcionamiento de las cantinas escolares, Gaceta de Madrid, no 11, (11-01-1912)

5 Véase R.O. de 22 de enero de 1921 por la que se crean tres plazas de Maestras para auxiliar los trabajos de la cantina. Gaceta de Madrid, no 35, (04-02-1921).

6 En este sentido se publicó en la Gaceta de Madrid: Orden de 8 de abril de 1927 por la que se concede audiencia a los representantes de la Fundación de El Ferrol, Gaceta de Madrid, no 125, (05-05-1927); R.O. número 1.215 de 16 de junio de 1930, Gaceta de Madrid, no 172, (21-06-1930).

7 R.O. número 2.127 de 29 de Octubre de 1930, Gaceta de Madrid, no 330, (26-11-1930).

8 Véase Decreto de 28 de agosto de 1931 sobre la integración de la cantina en la obra educativa de la Escuela, Gaceta de Madrid, no 241, (29-08-1931)

9 Al respecto véase las siguientes ordenes: Orden de 16 de noviembre de 1932 sobre la Fundación de la Cantina Escolar de Logroño, Gaceta de Madrid, no 328, (23-11-1932); Orden de 8 de febrero de 1935 sobre la Fundación denominada «Cantina Escolar Galbete», Gaceta de Madrid, no 40, (09-02-1935); Orden de 8 de febrero de 1935 sobre la Fundación «Cantina Escolar Mendía», Gaceta de Madrid, no 41, (10-02-1935).

10 Decreto de 27 de abril de 1935 por el que se crea en el Ministerio de Instrucción Pública y Bellas Artes una Comisión Central de colonias, cantinas y roperos escolares, Gaceta de Madrid, no 120, (30-04-1935).

11 Esta cifra aparece en un cartel de la época: PERIS, F. (1937), EI Gobierno radical-cedista dedicaba en 1935-36, 2.000.000 en cantinas escolares, el gobierno del Frente Popular dedica en 1937, 7.000.000, [Documento gráfico], Madrid, Gráficas Reunidas, UHP.

12 Decreto de 27 de abril de 1935; Decreto de 24 de mayo de 1935 por el que se estudia la organización de las instituciones complementarias de colonias, cantinas y roperos escolares, Gaceta de Madrid, no 146, (26-05-1935).

13 Véase la Orden de 26 de agosto de 1935 por la que se crea en toda Escuela Nacional de Primera enseñanza una Comisión protectora, Gaceta de Madrid, no 249, (06-09-1935).
14 Véase Orden de 3 de septiembre de 1936 sobre las aportaciones escolares para el monumento para doña María Cristina de Habsburgo, Gaceta de Madrid, no 248, (04-09-1936); Orden de 22 de septiembre de 1936 sobre las aportaciones escolares para el monumento para doña María Cristina de Habsburgo, Gaceta de Madrid, no 286, (12-10-1936).

15 Orden de 29 de septiembre de 1937 por la que se aprueba el gasto de referencia a la razón social «Hijos de M. Grases», Gaceta de la República, no 280, (07-10-1937).

16 Decreto de 24 de diciembre de 1937 por el que se concede un suplemento de crédito de 4.961 .000 pesetas al concepto «Subvención con destino a colonias, cantinas y roperos escolares», Gaceta de la República, no 360, (26-12-1937).

17 Para la elaboración de esta figura se han consultado las siguientes ordenes: Orden de 3 de diciembre de 1940 por la que se dispone la distribución del crédito asignado para Cantinas escolares a favor de los Centros de enseñanza que se detallan, BOE, no 353, (18-12-1940); Orden de 2 de diciembre de 1941 por la que se resuelven peticiones de auxilio para el sostenimiento de Cantinas y Comedores escolares, BOE, no 339, (05-12-1941); Resolución de 18 de diciembre de 1941 referente a las subvenciones ya otorgadas con destino a las Cantinas y Comedores escolares que se expresan y a la concesión de nuevo auxilio a las que se detallan, BOE, no 354, (20-12-1941); Orden de 26 de diciembre de 1941 concediendo a las Cantinas y Comedores escolares, que sostienen los centros y entidades que se citan, las subvenciones que se mencionan, BOE, no 362, (28-12-1941); Orden de 20 de julio de 1942 sobre concesión de subvenciones con destino a Cantinas y Comedores escolares, BOE, no 211, (30-07-1942); Orden de 14 de agosto de 1942 por la que se concede subvención para la organización y sostenimiento de las Cantinas y Comedores escolares de diversos grupos y centros de enseñanza primaria, $\mathrm{BOE}, \mathrm{n}$ - 234, (22-08-1942); Orden de 18 de noviembre de 1942 por la que se conceden subvenciones para Cantinas y Comedores escolares en diversos centros y entidades docentes, BOE, $\mathrm{n}$ ㅇ 333, (29-11-1942); Orden de 20 de diciembre de 1943 por la que se distribuyen 1.540 .000 pesetas de subvenciones por el concepto de Cantinas Escolares, BOE, no 357, (23-121943); Orden de 30 de diciembre de 1944 sobre distribución de crédito para cantinas escolares. BOE, $\mathrm{n}$ ำ10, (10-01-1945).

18 Ley de 17 de julio de 1945 sobre Educación Primaria, BOE, n으 199, (18-07-1945).

19 Decreto de 11 de enero de 1952 por el que se crea el Servicio de Protección de la Alimentación Escolar Infantil, BOE, n우 19, (19-01-1952)

20 Convenio de 7 de mayo de 1954 entre el Fondo de las Naciones Unidas para la Infancia y el Gobierno español, BOE, n을, (2111-1959)

21 Decreto de 11 de junio de 1954 por el que se crea la Comisión Interministerial para el Auxilio Internacional a la Infancia, BOE, no $194,(13-07-1954)$

22 Decreto 1788/1965, de 24 de junio, por el que se actualiza y estructura la Comisión Interministerial de Auxilio Internacional a la Infancia, BOE, no 160, (06-07-1965).

23 Al respecto consultar la Orden de 25 de Octubre de 1954 por la que se crea y dan normas para la actuación del Servicio Escolar 
de Alimentación, BOE, no 338, (4-12-1954) y la monografía de la Diputación de Castellón (1963).

24 Decreto de 13 de diciembre de 1954 por el que se amplía la composición y funciones de la Comisión Interministerial para el Auxilio Internacional a la Infancia, BOE, no 352, (18-12-1954).

25 Al respecto consultar el trabajo de Cenarro (2006) y el documento Normas ejecutivas para el desarrollo de la Ayuda Social Americana prevista en el Decreto de 13 de diciembre de 1954, BOE, no 45, (14-02-1955)

26 Esta afirmación se recoge en las Normas ejecutivas para el desarrollo de la Ayuda Social Americana prevista en el Decreto de 13 de diciembre de 1954.

27 Decreto de 21 de agosto de 1956 sobre el fomento de la fabricación de productos lácteos básicos para el crecimiento de la infancia, BOE, nㅇ 296, (22-10-1956).

28 Para la elaboración de esta figura se han consultado los documentos: Decreto-Ley de 24 de junio de 1955 sobre concesión de un crédito extraordinario de 100.000 .000 de pesetas para los gastos que origine la distribución en España de los artículos alimenticios de la Ayuda Social Americana, BOE, n 187, (06-071955); Ley de 26 de diciembre de 1958 por la que se concede un suplemento de crédito de 182.000 .000 de pesetas al Ministerio de Asuntos Exteriores para los gastos de distribución de la Ayuda Social Americana en España durante el año en curso, BOE, no 311, (29-12-1958); Ley 113/1960, de 22 de diciembre, por la que se concede un suplemento de crédito de 70.000.000 de pesetas al Ministerio de Asuntos Exteriores para satisfacer los gastos que ocasione la distribución de artículos alimenticios ejecutada por la Ayuda Social Americana, BOE, no 308, (24-121960); Ley 23/1962, de 21 de julio, por la que se concede un crédito extraordinario de 70.975.137,55 pesetas al Ministerio de Asuntos Exteriores para satisfacer las obligaciones que durante 1961 han originado las funciones encomendadas a la Comisión Interministerial de Auxilio Internacional a la Infancia, en relación con la Ayuda Social Americana y el Servicio Escolar de Alimentación, BOE, no 175, (23-07-1962); Ley 116/1964, de 16 de diciembre, por la que se concede un crédito extraordinario de 8.489.489 pesetas a Gastos de las Contribuciones y de diversos Ministerios para satisfacer gastos correspondientes al ejercicio de 1963 de la Gerencia de la Comisión Mixta de Productos Lácteos pro bienestar infantil y Cáritas Nacional Española, en ejecución de la Ayuda Social Americana, BOE, no 303, (18-121964); Ley 89/1966, de 28 de diciembre, de concesión de un suplemento de crédito de 120.000 .000 de pesetas, a «Gastos de las Contribuciones y de diversos Ministerios», con destino a satisfacer las obligaciones que en el presente ejercicio han de ser atendidas por la Comisión Interministerial de Auxilio Internacional a la Infancia, en relación con el Programa de Ayuda Social Americana y Productos Lácteos pro Bienestar Infantil y Social, BOE, no 311, (29-12-1966).

29 Orden de 27 de septiembre de 1959 por la que se crea la Comisión Mixta de Productos Lácteos pro Bienestar Infantil y Social, BOE, n 255, (24-10-1959).

30 Su composición y ampliación se recogió en las siguientes órdenes: Orden de 4 de diciembre de 1959 por la que se amplía la Comisión Interministerial de Auxilio Internacional a la Infancia, BOE, no 3, (04-01-1960); Orden de 13 de enero de 1960 por la que se amplía la Comisión Mixta de Productos Lácteos Pro-bienestar Infantil y Social, BOE, № 16, (19-01-1960).
31 El curso se convocó en la Orden de 10 de enero de 1962 sobre convocatoria de un Curso para Diplomados en Alimentación y Nutrición, y normas para la selección de los cincuenta y seis educadores participantes, BOE, no 17, (19-01-1962). Además, esta información ha sido confirmada y ampliada gracias a las entrevistas realizadas a varios informadores clave que participaron en este curso y que aportaron, junto con documentación impresa, numeroso material gráfico.

32 Al respecto se puede consultar el trabajo de Trescastro-López y Trescastro-López (2013) y la Orden de 24 de julio de 1962 por la que se crean Escuelas nacionales de Educación en Alimentación y Nutrición en régimen de Consejo Escolar Primario, BOE, no 192, (11-08-1962).

33 Orden de 10 de junio de 1963 por la que se crea, dentro de la Jefatura Central del Servicio Escolar de Alimentación y Nutrición, los Departamentos de: 1. Departamento de Educación en Alimentación y Nutrición. 2. Departamento de Complemento Alimenticio. 3. Departamento de Comedores Escolares y 4. Departamento de Programas, BOE, no 156, (01-07-1963); Orden de 18 de junio de 1963 por la que se convoca concurso de méritos para proveer, en propiedad, las Jefaturas de los Departamentos de Educación en Alimentación y Nutrición, del Servicio Escolar de Alimentación y Nutrición, BOE, no 159, (04-07-1963); Orden de 22 de octubre de 1963 por la que se nombran Jefes de Departamentos del Servicio Escolar de Alimentación y Nutrición a los señores que se indican, BOE, no 293, (07-12-1963).

34 Dolores Prados fue directora del departamento de actividades educativas del SEAN e inspectora de Enseñanza Primaria. Véase el trabajo Prados (1968).

35 Real Decreto 808/1978, de 27 de marzo, sobre transferencia de las funciones de la Comisión Interministerial Pro Bienestar Infantil y Social (CIBIS) al Fondo Nacional de Asistencia Social, BOE, no 98, (25-04-1978).

36 Decreto 1788/1965, de 24 de junio, por el que se actualiza y estructura la Comisión Interministerial de Auxilio Internacional a la Infancia, BOE, no 160, (06-07-1965).

37 Orden de 27 de octubre de 1965 por la que se convoca concurso de méritos para seleccionar 30 Maestros nacionales que participarán en el II Curso para Diplomados de Educación en Alimentación y Nutrición, BOE, no 270, (11-11-1965).

38 Acerca de la historiografía que se ha ocupado de cuestiones antropométricas, véase el trabajo de Martínez Carrión (2011) y el artículo de Ballester y Perdiguero (2000).

39 Orden de 12 de enero de 1973 por la que se adscribe la dirección de los programas de la Comisión Interministerial Pro Bienestar Infantil y Social a la Dirección General de Política Interior y Asistencia Social, BOE, no 17, (19-01-1973).

40 Orden de 5 de julio de 1973 por la que se declaran disueltos el Consejo Escolar Primario de Maestros Auxiliares Especializados en Organización de Servicios Escolares y el creado por las Escuelas de Educación en Alimentación y Nutrición, BOE, no 165, (11-07-1973).

41 Real Decreto 808/1978.

42 Orden de 5 de junio de 1978 por la que se aprueba el Plan de Inversiones del Fondo Nacional de Asistencia Social para el ejercicio de 1978, BOE, no 161, (07-07-1978). 


\section{BIBLIOGRAFÍA}

Agencia Española de Seguridad Alimentaria (2005), La Estrategia NAOS. Estrategia española para la nutrición, actividad física y prevención de la obesidad, Madrid, Ministerio de Sanidad y Consumo.

Altava, Vicente (1957), Crecimiento y alimentación de los castellonenses, Castellón de la Plana, Excma. Diputación de Castellón.

Ballester, Rosa (1994), "La salud del niño en edad escolar: los inicios de la inspección médico-escolar en España”. En: La escuela y los maestros, 1857-1970, Alicante, Generalitat Valenciana-Fundación cultural de la CAM-Instituto de Cultura Juan Gil Albert de la Diputación de Alicante, pp. 111-124.

Ballester, Rosa y Perdiguero, Enrique (2000), "Los estudios sobre crecimiento humano como instrumento de medida de la salud de los niños españoles (1900-1950)", Áreas: Revista internacional de ciencias sociales, 20, pp. 161-170.

Barciela, Carlos (2000), La Ayuda Americana a España (1953-1963), Alicante, Universidad.

Barona, Josep Lluís (2006), "Defining the "Children's Problem» in Spain, 1900-1930. Political Initiatives and Ideological Debates". En: Andresen, Astri; Gronlie, Tore y Ryymin, Teemu (eds.), Science, Culture, and Politics. European Perspectives on Medicine, Sickness and Health: conference proceedings, Bergen, Rokkansenteret, Stein Rokkan Centre for Social Studies, pp. 15-29.

Barona, Josep Lluís y Bernabeu-Mestre, Josep (2008), La salud y el Estado. El movimiento sanitario internacional y la administración española (1851-1945), València, Publicacions Universitat de València.

Barona, Josep Lluís (2012), "Nutrition, Public Health and Education". En: Barona, Josep Lluís, From Hunger to Malnutrition: the political economy of scientific knowledge in Europe, 1918-1960, Brussels, Peter Lang, pp.295-326.

Bernabeu-Mestre, Josep et al. (2011), "El contexto histórico de la transición nutricional en España”. En: Bernabeu-Mestre, Josep y Barona, Josep Lluís (eds.), Nutrición, salud y sociedad. España y Europa en los siglos XIX y XX, València, Seminari d'Estudis sobre la Ciència/Publicaciones de la Universidad de Valencia, pp. 185-208.

Bernabeu-Mestre, Josep, Trescastro-López, Eva y Galiana-Sánchez, M.a Eugenia (2011), "La divulgación radiofónica de la alimentación y la higiene infantil en la España de la Segunda República (1933-1935)", Salud Colectiva, 7 (Supl 1), pp. S49-S60.

Casado, Demetrio (1967), Perfiles del hambre, Madrid, Edicusa.

Cenarro, Ángela (2006), La sonrisa de Falange. Auxilio Social en la guerra civil y en la posguerra, Barcelona, Crítica.

De Palacios, Juan Manuel (1969), Información sobre el Programa de Educación en Alimentación y Nutrición en España, Madrid, Gobierno Español (CIBIS)-FAO-UNICEF.

Del Cura, María Isabel y Huertas, Rafael (2007), Alimentación y enfermedad en tiempos de hambre. España, 1937-1947, Madrid, CSIC.
Diputación de Castellón. Servicio Escolar de Alimentación y Nutrición (1963), Alimentación y Nutrición, Castellón, Diputación de Castellón.

Esplugues-Pellicer, Josep Xavier y Trescastro-López, Eva María (2012), "El Programa de Educación en Alimentación y Nutrición: cuando la alimentación española era satisfactoria (1961-1972)". En: Gracia, Mabel (ed.), Alimentación, salud y cultura: encuentros interdisciplinares, Tarragona, Publicaciones URV (Colección de Antropología Médica), pp. 423-440.

García, Abraham y López, Consuelo (1990), “Nuestra experiencia en el programa EDALNU", Nutrición Clínica y Dietética Hospitalaria, 10 (4), pp. 137-144.

Hendrich, Harry (1992), "Child labour, medical capital and the school medical service". En: Cooter, Roger (ed.), In the name of the child: health and welfare, 1880-1940, London, Routledge, pp.45-71.

Hernández, Mireia, Hernández, Carles y Sanromà, Joan (2005), Celestina Vigneaux. Les cantines escolars a Barcelona i la renovació pedagògica a l'escola pública, Barcelona, Ajuntament de Barcelona, Abadia de Montserrat.

López, Consuelo (1972), "El programa español de Educación en Alimentación y Nutrición”, Revista de Sanidad e Higiene Pública, 46 (11-12), pp. 951-958.

López, Consuelo (1973), "Resumen de las tareas realizadas a través del Programa de Educación en Alimentación y Nutrición (Programa EDALNU)", Revista de Sanidad e Higiene Pública, 47 (6), pp. 559-578.

López, Consuelo (1987), “Los problemas de nutrición aplicada: EI Programa EDALNU”, Nutrición Clínica y Dietética Hospitalaria, VII (1), pp. 62-64.

López, Consuelo et al. (1998), Apuntes del curso de fundamentos de Nutrición Comunitaria, Madrid, UNED.

Martínez Carrión, José Miguel (2011), “El estado nutricional en la Europa contemporánea. Una visión desde la historia antropométrica". En: Bernabeu-Mestre, Josep y Barona, Josep Lluís (eds.), Nutrición, salud y sociedad. España y Europa en los siglos $X I X$ y XX, València, Seminari d'Estudis sobre la Ciència/Publicaciones de la Universidad de Valencia, pp. 93-131.

Perdiguero, Enrique (comp.)(2004), Salvad al niño. Estudios sobre la protección a la infancia en la Europa mediterránea a comienzos del siglo XX, Valencia, Seminari d’Estudis sobre la Ciència.

Prados, Dolores (1968), El comedor escolar, Madrid, Ministerio de Educación y Ciencia, Dirección General de Enseñanza Primaria.

Rigolfas, Rita; Padró, Laura y Cervera, Pilar (2010), Educar en la alimentación y la nutrición, Barcelona, Tibidabo Ediciones.

Sánchez-Granjel, Mercedes (2003), Las Hurdes, el país de la leyenda: entre el discurso ilustrado y el viaje de Alfonso XIII, Lleida, Milenio. 
Scott, Marjorie (1954), La alimentación escolar y su influencia en la nutrición del niño, Roma, FAO.

Serra-Majem, Luís y Bautista Castaño, Inmaculada (2007), "La nutrición en España". En: Como vivíamos: alimentos y alimentación en la España del siglo XX, Barcelona, Lunwerg, pp. 177-195.

Servicio Escolar de Alimentación (1955), Documenta. Cuaderno $n$ 요, Madrid, Dirección General de Prensa.

Trescastro-López, Eva M.ạ; Bernabeu-Mestre, Josep y GalianaSánchez, M. a Eugenia (2011), "Nutrición comunitaria y alimentación escolar en España: el ejemplo de las cantinas escolares de la Asociación de Caridad Escolar de Madrid (1901-1927)", Revista Española de Nutrición Comunitaria, 17 (4), pp. 206-212.

Trescastro-López, Eva M.a; Galiana-Sánchez, M.ạ Eugenia y Bernabeu-Mestre, Josep (2012), “El Programa de Educación en
Alimentación y Nutrición (1961-1982) y la capacitación de las amas de casa como responsables del bienestar familiar", Nutrición Hospitalaria, 27 (4), pp. 955-963.

Trescastro-López, Eva M.a y Trescastro-López, Silvia (2013), "La educación en alimentación y nutrición en el medio escolar: el ejemplo del Programa EDALNU", Revista Española de Nutrición Humana y Dietética, 17 (2), pp. 84-90.

Vivanco, Francisco (1965), “La educación sanitaria en materia de alimentación". En: Sociedad Española de Higiene y Medicina Social (ed.), Problemas de educación sanitaria, Madrid, Gráficas Valera S.A., pp. 81-98.

Vivanco, Francisco; Palacios, Juan Manuel y García, Abraham (1984), Alimentación y nutrición. 2a ed., Madrid, Ministerio de Sanidad y Consumo. 